\title{
A NOTE ON $z$-CLOSED PROJECTIONS
}

\author{
N. NOBLE ${ }^{1}$
}

All hypothesized spaces are assumed to be completely regular Hausdorff. Recall that the projection $\pi_{X}: X \times Y \rightarrow X$ is called $z$-closed if it maps zero sets to closed sets. This notion has recently been used to give conditions under which $\nu(X \times Y)=\nu X \times \nu Y(\nu X$ denotes the Hewitt-Nachbin realcompactification of $X)-[\mathbf{C N}]$; to characterize products $X \times Y$ for which the product uniformity is fine- $\left[\mathbf{N}_{1}\right]$ and $[\mathbf{H}]$; and to prove exponential laws and Ascoli Theorems- $\left[\mathbf{N}_{2}\right]$. Some further applications will be given in $\left[\mathbf{N}_{3}\right]$. In this note, using the more general notion of relatively $z$-closed projections, we give some new conditions under which a projection on a product will be $z$-closed.

Throughout, $\beta X$ denotes the Stone-Čech compactification of $X, I$ denotes the unit interval, and $C(X \times Y, I)$ denotes the set of continuous functions from $X \times Y$ to $I$. For $f$ in $C(X \times Y, I)$, the function which maps $x$ to $\sup \{f(x, y): y \in Y\}$ will be denoted by $\sup _{Y} f$. We will consider $\sup _{Y}$ as a function on $C(X \times Y, I)$. Recall that a space in which each $\boldsymbol{\aleph}$-fold intersection of open sets is open is called $\boldsymbol{\aleph}$-discrete, and a space in which each family of nonempty disjoint open sets which has no cluster point has cardinality less than $\boldsymbol{N}$ is called pseudo- $\boldsymbol{\aleph}$-compact. Pseudo- $\boldsymbol{\aleph}_{0}$-compact $=$ pseudocompact and $\aleph_{0}$-discrete $=P$-space. Our first result gives a condition equivalent to the condition: $\pi_{X}$ is $z$-closed. The implication (i) $\Rightarrow$ (ii) with $Y$ pseudocompact was established in $\left[\mathrm{HM}_{0}\right]$ and generalizes [F, Lemmas 1.1 and 1.3]. The implication (ii) $\Rightarrow(\mathrm{i})$, and its proof, was pointed out to us, jointly, by W. W. Comfort and A. W. Hager.

THEOREM 1. The following two conditions are equivalent:

(i) $\pi_{X}: X \times Y \rightarrow X$ is z-closed;

(ii) $\sup _{Y}(C(X \times Y, I))=C(X, I)$.

Proof. (i) $\Rightarrow$ (ii). Let $f$ be any function in $C(X \times Y, I)$. Then $\sup _{Y} f$ is lower semicontinuous; to see that $\sup _{Y} f$ is upper semicontinuous, note that for any point $a$ in $I$,

$$
\left(\sup _{Y} f\right)^{-1}([a, 1])=\bigcap_{n=1}^{\infty} \pi_{X} f^{-1}([a-1 / n, 1])
$$

Received by the editors August 22, 1968.

1 Work on this note was partially supported by NASA Grant no. 93-6. 
which is closed. Thus $\sup _{Y} f$ is continuous, so $\sup _{Y}(C(X \times Y, I))$ $\subseteq C(X, I)$. The reverse inclusion is trivial.

(ii) $\Rightarrow$ (i): Let $Z \subseteq X \times Y$ be a zero set, say $Z=f^{-1}(0)$ with $f$ in $C(X \times Y, I)$, and let $x_{0}$ be any point in $X \backslash \pi_{X} Z$. For $g$ defined by the rule $g(x, y)=\max \left\{f(x, y) / f\left(x_{0}, y\right), 1\right\}, g$ is in $C(X \times Y, I)$ so $\sup _{Y} g$ is continuous. Since $\sup _{Y} g\left(x_{0}\right)=1$ while $\sup _{Y} g\left(\pi_{X} Z\right)=0$, it follows that $x_{0}$ is not in the closure of $\pi_{X} Z$.

For $X^{\prime} \subseteq X$, a subset of $X^{\prime}$ is called a relative zero set (of $X^{\prime}$ in $X$ ) if it is the intersection with $X^{\prime}$ of a zero set of $X$. We call the projection $\pi_{X^{\prime}}: X^{\prime} \times Y \rightarrow X^{\prime}$ relatively $z$-closed (with respect to $X \times Y$ ) if it maps the relative zero sets of $X^{\prime} \times Y$ in $X \times Y$ to closed sets. Relatively $z$-closed projections were introduced, and used, in $\left[\mathbf{N}_{2}\right]$, where it was shown that they act almost like $z$-closed projections. Thus the following is to be expected.

THEOREM 2. For $X^{\prime} \subseteq X$, the following two conditions are equivalent:

(i) $\pi_{X^{\prime}}: X^{\prime} \times Y \rightarrow X^{\prime}$ is relatively $z$-closed;

(ii) For each $f$ in $C(X \times Y, I)$, the restriction of $\sup _{Y} f$ to $X^{\prime}$ is continuous.

Proof. By the obvious generalization of the proof of Theorem 1 .

Recall that a subset, $X^{\prime}$, of $X$ is called relatively pseudocompact (in $X$ ) if each continuous real-valued function on $X$ is bounded on $X^{\prime}$. We will need the following results, which were established in $\left[\mathbf{N}_{2}\right]$ :

Lemma. (i) If $X^{\prime} \subseteq X$ and $\pi_{X^{\prime}}: X^{\prime} X Y \rightarrow X^{\prime}$ is relatively z-closed, then either $Y$ is pseudocompact or $X^{\prime}$ is a $P$-space.

(ii) Let $X^{\prime} \subseteq X$ and suppose $X^{\prime}$ is relatively pseudocompact and not discrete. Then $\pi_{X^{\prime}}: X^{\prime} \times Y \rightarrow X^{\prime}$ is relatively $z$-closed if and only if $X^{\prime} \times Y$ is relatively pseudocompact.

(iii) $X \times Y$ is pseudocompact for each pseudocompact space $X$ if and only if $X^{\prime} \times Y$ is relatively pseudocompact in $X \times Y$ whenever $X^{\prime}$ is relatively pseudocompact in $X$.

We call a space, $X$, a $b_{R}$-space if a real-valued function on $X$ (equivalently a function into $I$ ) is continuous whenever its restriction to each relatively pseudocompact subset of $X$ is continuous. If $X$ is a $b_{R}$-space and each relatively pseudocompact subset of $X$ is discrete, then each real-valued function on $X$ is continuous so $X$ is discrete. In particular, a $P$-space is a $b_{R}$-space if and only if it is discrete.

Theorem 3. Let $X$ be a $b_{R}$-space and let $Y$ be arbitrary. Then $\pi_{X}$ : $X \times Y \rightarrow X$ is z-closed if and only if one of the following two conditions is satisfied: 
(i) $X$ is discrete;

(ii) For each relatively pseudocompact $X^{\prime} \subseteq X, X^{\prime} \times Y$ is relatively pseudocompact in $X \times Y$.

Proof. If $X$ is discrete, then $\pi_{X}$ is closed, hence $z$-closed. Suppose (ii) holds; by Theorem 1 to show that $\pi_{X}$ is $z$-closed it suffices to show, for any $f$ in $C(X \times Y, I)$, that $\sup _{Y} f$ is continuous. But by (ii), the lemma, and Theorem $2, \sup _{Y} f$ is continuous on each relatively pseudocompact subset of $Y$. Since $X$ is a $b_{R}$-space, it follows that $\sup _{Y} f$ is continuous.

Conversely, suppose $\pi_{X}: X \times Y \rightarrow X$ is z-closed. Then either (i) holds or (by the lemma) $Y$ is pseudocompact. But if $Y$ is pseudocompact, then the product of $Y$ with each discrete relatively pseudocompact subset of $X$ is relatively pseudocompact since the discrete relatively pseudocompact subsets of $X$ are finite. Since by the lemma the product of $Y$ with each nondiscrete relatively pseudocompact subset of $X$ is relatively pseudocompact (because $\pi_{X^{\prime}}: X^{\prime} \times Y \rightarrow X^{\prime}$ is relatively $z$-closed for each $X^{\prime} \subseteq X$ ) it follows that (ii) holds.

Define $b_{R}^{*}$-spaces analogously to $b_{R}$-spaces, replacing in the definition relatively pseudocompact subsets by those subsets whose product with each pseudocompact space is relatively pseudocompact in the product. (An intrinsic characterization of such subsets is given in $\left[\mathbf{N}_{3}\right]$.) The spaces defined in a similar manner but using compact subsets are called $k_{R}$-spaces. Obviously each $k_{R}$-space is a $b_{R}^{*}$-space.

Corollary 1. If $X$ is a $b_{R^{-}}^{*}$ pace, then for each pseudocompact space $Y \pi_{X}: X \times Y \rightarrow X$ is z-closed.

CoRollary 2. The product of $X$ with each pseudocompact space is pseudocompact if and only if $X$ is a pseudocompact $b_{R}^{*}$-space.

Proof. By, for instance, [ $\mathbf{N}_{1}$, Theorem 3.4], $X \times Y$ is pseudocompact if $X$ is pseudocompact and $\pi_{X}: X \times Y \rightarrow X$ is $z$-closed. Thus the product of a pseudocompact $b_{R}^{*}$-space with a pseudocompact space is pseudocompact. The remaining implication is trivial.

As a description of the class of spaces whose product with each pseudocompact space is pseudocompact, Corollary 2 is vastly inferior to the description given in $[F]$. It does, however, make obvious the fact (proved in $\left[\mathbf{N}_{4}\right]$ ) that pseudocompact $k_{R}$-spaces are in this class.

Corollary 3. A space $Y$ has the property that $\pi_{X}: X \times Y \rightarrow X$ is $z$-closed for each $b_{R}$-space $X$ if and only if the product of $Y$ with each pseudocompact space is pseudocompact (i.e., iff $Y$ is a pseudocompact $b_{R}^{*}$-space). 
Proof. Theorem 2 and the lemma.

If $X$ is assumed to be $\langle\boldsymbol{\aleph}$-discrete but not $\boldsymbol{\aleph}$-discrete, if "relatively pseudocompact" is replaced by "relatively pseudo- $\boldsymbol{\aleph}$-compact" (see $\left[\mathbf{N}_{2}\right]$ for definitions of this term) and if $b_{R}$-spaces and $b_{R}^{*}$-spaces are replaced by the appropriate classes of spaces, then the statements and proofs of these results generalize in the obvious manner.

Finally, we pose a question. Is the converse of Corollary 1 or 3 true? I.e., are the classes of $b_{R}^{*}$-spaces and $b_{R}$-spaces the largest for which these results can hold? (The notions of $b_{R}^{*}$-spaces and $b_{R}$-spaces are sufficiently gruesome that one might expect that this is the case.) In this context, note the question posed in [FF]: If for each countably compact space $Y$, the projection $\pi_{X}: X \times Y \rightarrow X$ is closed (maps closed sets to closed sets), must $X$ be a subspace of a sequential space? The converse is true, and $b_{R}$-spaces are a natural analogue of sequential spaces in this context

\section{REFERENCES}

[CN] IV. Comfort and S. Negrepontis, Extending continuous functions on $X \times Y$ to subsets of $\beta X \times \beta Y$, Fund. Math. 59 (1966), 1-12.

[F] Z. Frolik, The topological product of two pseudocompact spaces, Czechoslovak. Math. J. 10 (1960), 339-349.

[FF] I. Fleischer and S. P. Franklin, On compactness and projections, (to appear).

[G] I. Glicksberg, Stone-Čech compactifications of products, Trans. Amer. Math. Soc. 90 (1959), 369-382.

[GJ] L. Gillman and M. Jerison, Rings of continuous functions, Van Nostrand, Princeton, N. J., 1960.

[H] A. Hager, Projections of zero-sets (and the fine uniformity on a product), Trans. Amer. Math. Soc. 140 (1969), 87-94.

[HM] A. Hager and S. Mrowka, (a) Compactness and the projection mapping from a product space, Notices Amer. Math. Soc. 12 (1965), 368 (Abstract 65T-167). (b) Unpublished manuscript.

$\left[\mathrm{N}_{1}\right]$ N. Noble, Products with closed projections, Trans. Amer. Math. Soc. 140 (1969), 381-391.

$\left[\mathrm{N}_{2}\right]-$, Ascoli theorems and the exponential map, Trans. Amer. Math. Soc. (to appear).

$\left[\mathbf{N}_{3}\right]$ - Products of quotient maps and spaces with weak topologies, (to appear).

$\left[\mathrm{N}_{4}\right] \longrightarrow$, Countably compact and pseudocompact products, Czechoslovak. Math. J. (to appear).

\section{Clark University}

